\title{
Model of multicomponent micro-Doppler signal in environment MatLab
}

\author{
Alexander Kucheryavenko, ${ }^{1,}$, Valentine Fedosov ${ }^{1}$ \\ ${ }^{1}$ Institute Radioengineering Systems and Control, Southern Federal University, 344006, Rostov-on-Don, Russia
}

\begin{abstract}
The article illustrates the problem of measuring the speed glider component targets in the presence of a turboprop effect of the reflected signal in a pulse-Doppler radar, proposed a model turboprop signal component and an algorithm for its suppression
\end{abstract}

\section{Turbine component}

Modern radar systems make high demands on the processing of the received signals. This is especially true echo signals from aerodynamic objects.Therefore, the task of shaping the radiolocation portraits of aerodynamic objects are becoming increasingly important against the backdrop of expanding opportunities and increasing the performance of systems for digital processing.Modern methods of radio vision and formed on their basis radar portraits of aerodynamic objects can be used for [1]: recognition of classes or types of aerodynamic objects, including the air corridors of commercial airlines; the meteorologic formations, flocks of birds; providing dispatchers with information about the true average angle of the setting on landing of the aircraft in a strong crosswind and the availability of damages in hydroaerodynamics equipment.

When analyzing the echo signals in radar systems, the dynamics of the reflecting surface of the aerodynamic object, arising due to scattering from the rotating elements of the design, is evident in the spectrum of the reflected signal [2].

The phenomenon of turbo-propeller effect is expressed by the presence in the spectrum of the echo signal along with the body component additional spectral components, called turbine components.

The presence of the turbine component in the echo signal caused by the moving parts of the aerodynamic object [2]. The components of the spectrum caused by turboprop effect can mask the signal from a stationary part of the object and degrade the image on the radar. Therefore, the separation the parts of the spectrum caused by the stationary part of the object from the spectrum caused by moving, rotating or vibrating parts and is of interest for the research.

The fixed part of the aerodynamic object, on the radar picture is presented by glider leaves and rotating and vibrating parts of the object cause the turbine components.

Reflecting from a moving aerodynamic object, radar signals acquires additional modulation this is a

\footnotetext{
"Corresponding author: zetsu85@gmail.com
}

manifestation of the turboprop effect. This is due to the presence in the composition of objects rotating elements (the turbine blades and compressor blades of the propellers).Secondary Doppler portrait as a spectrum of the reflected signal and an integral part of the twodimensional radar image of the object may be distorted by the presence of additional harmonic components not related to the geometric characteristics of the airframe aerodynamic object.

The effects of turbine effects on the spectrum reflected from the aerodynamic object signal is expressed in the appearance of additional harmonics. The spectrum is always linear.

When processing signals from aerodynamic objects in the background noise one of the key problems is the allocation of the individual components of the useful signal [2].

In pulsed radar stations for obtaining a spectral Doppler portraits uses the values of the amplitudes and phases of radio pulses reflected from the aerodynamic object.This is equivalent to sampling the continuous reflected signal with a certain pulse repetition frequency.

\section{Algorithm for suppressing the turboprop effect}

It seems appropriate to develop the algorithm for suppression of turbine effect of the radar signal in pulseDoppler radar system. This algorithm in pulse Doppler radar system can be based on the difference of the Doppler increment frequency for a body component of the spectrum of the echo signal and the modulation frequency due to the turbine effect, and the analysis of changes in the time of full amplitude and instantaneous phase of the signal propriety of the turbine effect [3].To develop algorithm of suppression of turbine effect of the echo signal from the aerodynamic object in a pulsed-Doppler radar system, following parameters should be specified: glider aerodynamic speed of the object; the wavelength of the probing signal radar station; the distance to the aerodynamic object; an effective dissipation surface of the 
object; Doppler frequency of the turbine component; the level of the side components of the output signal relative to the carrier [3].

The algorithm should work with a priori unknown speed of rotation of the rotor or turbine rotor.

The comparison of the Doppler frequency increment for a body component of the spectrum and the modulation frequency due to the turbine effect, shows their essential difference, therefore, the time variation of the amplitude and the instantaneous phase of the full turbine effect is significantly slower than change these characteristics for a body component of the spectrum of the envelope of the echo signal.

Similarly, the change of the envelope there is a component unchanged in the process of formation of reflections from a body component of aerodynamic object and component, with the law changes over time, close to the law of change of the component of the total instantaneous phase, due to turbine effect.

Thus the full term of the instantaneous phase for a body component varies in time according to a linear law, and the summand corresponding to a turbine effect to a law close to harmonic, resulting in the appearance of the lateral components of the spectrum of the echo signal.

\section{Development of models}

As test signals it's necessary to develop a model of response signal from the aerodynamic object and the model of interference, which is a turboprop effect [3].

As a test signal it is recommended to use a bundle of linearly frequency modulated pulses.

Linear frequency modulated waveforms are used in radar as the method of formation and processing of the probe pulse. Application of linear frequency modulated signal allows to increase the measurement accuracy of radar.

The instantaneous value of the transmitted signal represented by the formula:

$$
\mathrm{s}(t)=\sum_{\mathrm{k}=1}^{\mathrm{N}} \mathrm{A} \cos \left(\omega t+\frac{\alpha t^{2}}{2}\right), t \in\left[\mathrm{t}_{\mathrm{u}}\right],
$$

where $\mathrm{A}$ is the pulse amplitude, $\omega$ - frequency filling pulse, $\alpha$ is the speed of linear frequency changes inside pulse $\mathrm{t} \_$и - pulse duration.

The instantaneous value of the received signal represented by the formula:

$$
\begin{gathered}
\mathrm{s}(t)=\sum_{\mathrm{k}=1}^{\mathrm{N}} \mathrm{A}\left[1+\mathrm{M} \cos \left(\Omega_{\mathrm{T}} t\right) \mathrm{s}_{\mathrm{k}}(t)\right] \\
\mathrm{s}_{\mathrm{k}}(\mathrm{t})=\sum_{\mathrm{k}=1}^{\mathrm{N}} \mathrm{A} \cos \left(\omega \mathrm{t}+\frac{\alpha \mathrm{t}^{2}}{2}+\mathrm{m}_{\mathrm{T}} \cos \left(\Omega_{\mathrm{T}} \mathrm{t}\right)\right),
\end{gathered}
$$

where $t \in\left[\mathrm{t}_{\mathrm{u}}\right], \mathrm{M}-$ the modulation ratio, $\Omega_{\mathrm{T}}-$ the frequency of the turbine component, $\mathrm{m}_{\mathrm{T}}$ - the modulation index of the turbine component.

Amplitude modulation occurs due to changes in effective surface scattering of the rotating blades of the turbine, and angular modulation due to the speed of rotation of these blades [3].

The optimum detector for coherent packets of radio pulses on a background of white noise is a serially connected: consistent with the packet filter, a detector and a threshold device. Complex frequency response (CFC) harmonized filter

$$
\mathrm{k}_{\mathrm{c}}(\mathrm{j} \omega)=\dot{\mathrm{S}}(\mathrm{j} \omega) \mathrm{e}^{-\mathrm{i} \omega \mathrm{T}}=\dot{\mathrm{S}}_{0}(\mathrm{j} \omega) \dot{\mathrm{S}}_{\mathrm{n}}(\mathrm{j} \omega) \mathrm{e}^{-\mathrm{i} \omega \mathrm{T}}
$$

is the complex conjugate function of the spectra of the bundles $\mathrm{S}(\mathrm{j} \omega)$.

Here $\mathrm{T}-$ is the delay time of the signal in the filter; $\dot{S}_{0}(j \omega)$ - single pulse spectrum; $\dot{S}_{n}(j \omega)-$ spectrum of the bundle of $\delta$-pulses.

The second equality in formula (4) makes it possible to divide the processing of the reflected signal during the period and between periods.

The task of signal detection against the background interference is correlated with the energy spectrum $\mathrm{G}_{\mathrm{n}}(\omega)$ can be lead to the problem of signal detection against the background of white noise, if you first carry out a "whitewash" interference, since the interference is fed to the input of the detector to mix with the white noise of spectral density N0.

The "whitewash" filter must have a complex frequency response

$$
\mathrm{K}_{\mathrm{oб}}(\mathrm{j} \omega)=\frac{\mathrm{K}_{0}}{\sqrt{\mathrm{G}_{\mathrm{n}}(\omega)+\mathrm{N}_{0}}} \mathrm{e}^{\mathrm{j} \varphi(\omega)},
$$

where K0 - constant; $\varphi(\omega)$ - arbitrary phase-frequency characteristic, which we believe zero.

Then the power spectral density of the mixture of the interference noise at the output of the filter

$$
\mathrm{G}_{\text {вых }}(\omega)=\left[\mathrm{G}_{\mathrm{n}}(\omega)+\mathrm{N}_{0}\right]\left|\mathrm{K}_{\text {об }}(\mathrm{j} \omega)\right|^{2}=\mathrm{K}_{0}^{2} .
$$

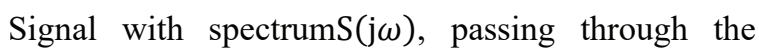
"whitewash" filter acquires as a result of filtering facilities $\mathrm{K}_{\text {об }}(\mathrm{j} \omega) \mathrm{S}(\mathrm{j} \omega)$, therefore, the matched filter must have a complex frequency response

$$
\mathrm{K}_{\mathrm{c} \phi}(\mathrm{j} \omega)=\dot{\mathrm{K}}_{\mathrm{o \sigma}}(\mathrm{j} \omega) \dot{\mathrm{S}}(\mathrm{j} \omega) \mathrm{e}^{-\mathrm{i} \omega \mathrm{T}}=\frac{\mathrm{K}_{0} \dot{\mathrm{S}}(\mathrm{j} \omega) \mathrm{e}^{-\mathrm{i} \omega \mathrm{T}}}{\sqrt{\mathrm{G}_{\mathrm{n}}(\omega)+\mathrm{N}_{0}}} .
$$

The complex frequency response of series-connected whitewash and coherent filters

$$
\mathrm{K}_{\mathrm{o \Pi T}}(\mathrm{j} \omega)=\frac{1}{\mathrm{G}_{\Pi}(\omega)+\mathrm{N}_{0}} \dot{\mathrm{S}}(\mathrm{j} \omega) \mathrm{e}^{-\mathrm{i} \omega \mathrm{T}}
$$

is the complex frequency response of an optimal filter of the detector quasideterminants signal on the background of correlated noise.

The expression corresponds to the series connected filter rejected passive interference with the complex frequency response

$$
\mathrm{K}_{\mathrm{o \Pi T}}(\mathrm{j} \omega)=\frac{1}{\mathrm{G}_{\Pi}(\omega)+\mathrm{N}_{0}}
$$

normal and agreed only with the signal filter

$$
\mathrm{K}_{\mathrm{c}}(\mathrm{j} \omega)=\dot{\mathrm{S}}(\mathrm{j} \omega) \mathrm{e}^{-\mathrm{i} \omega \mathrm{T}}
$$

Thus, the structural scheme of the optimal detector of known packs of samples consists of decorrelation noise, the unit weight of the accumulation and threshold device. 
The actual sequence of radio pulses can be converted into a sequence of discrete samples by applying a matched filter followed by the temporal discretization [4].

If the initial phase of the burst pulse is unknown, the weighting processing is carried out in two quadrature channels, followed by the Association of quadratures.

Under unknown Doppler frequency of the aerodynamic object blocks matched filtering and the weight savings become multi-channel frequency. The number of frequency channels is determined by the resolution in frequency and a frequency spectrum.

The most common kind of disturbing effects in the communication channel is additive white Gaussian noise [4].

Based on these findings, we propose the following algorithm to reduce the influence of the turbine effect, comprising the following operations:

1. determination of the dependence of the modulus of the complex envelope and its argument in time within the counts of the bundle of the reflected signal after applying matched filtering in range;

2. the smoothing argument of the complex envelope along the axis of times each element of a range, it is necessary to use polynomial smoothing above 20 order;

3 . the use of counts of the argument for smooth functions when restoring the complex envelope after the removal of the turbine effect on the argument in time. Modifying an argument caused by a turbine effect will be eliminated;

4. similarly, the smoothing module envelope in time and use the results of smoothing for the recovery module discrete values of the smoothed functions in time for each resolution element in range;

5. perform fast Fourier transformation on each element of a range.

This algorithm requires validation in modeling the problem of suppression of turboprop effect for a simple model of the echo signal.

Modeling complex envelope of the echo from a single object by the method of temporal smoothing provides full suppression of the turbine effect of a single source located at a constant distance.

\section{Simulation program}

To test the algorithm of suppression turboprop effect was applied to the test signals.

As the test signal the bundle of linear frequency modulated pulses have been taken.

The timing diagram of the emitted pulse packet is shown in figure 1.

This signal represents an even number of pulses with rectangular envelope and the frequency filling, which is a linearly frequency modulated radio signal.

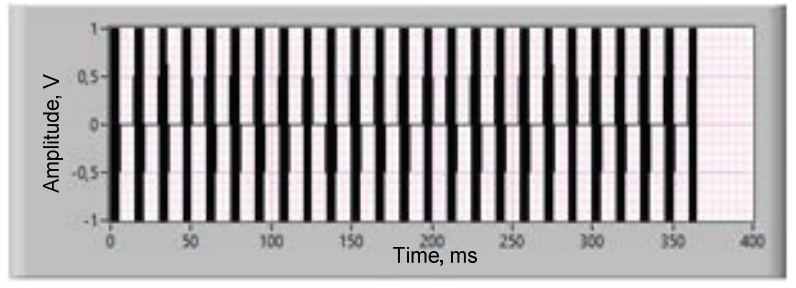

Fig. 1. Time diagram of the emitted burst of pulses.

The timing diagram of the envelope of the burst pulse is shown in figure 2. Obviously, the rectangular shape of the signals.

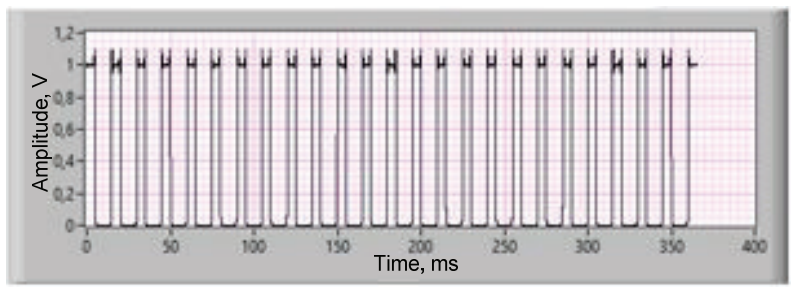

Fig. 2. Timing diagram of the envelope of the burst.

The spectrum of packets of pulses is presented in figure 3 .

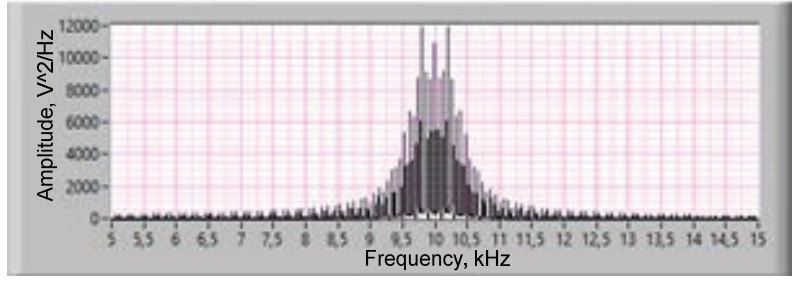

Fig. 3. The spectrum of the emitted burst of pulses.

This spectrum represents a typical spectrum of the linear frequency modulated signal.The timing diagram of the emitted pulse packet after applying matched filtering is presented in figure 4 .

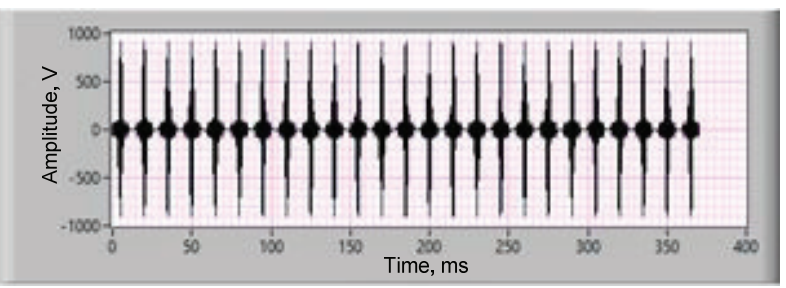

Fig. 4. Timing diagram of the emitted burst after consistent filtering.

The timing diagram of the envelope of the burst pulse after matched filtering is shown in figure 5.

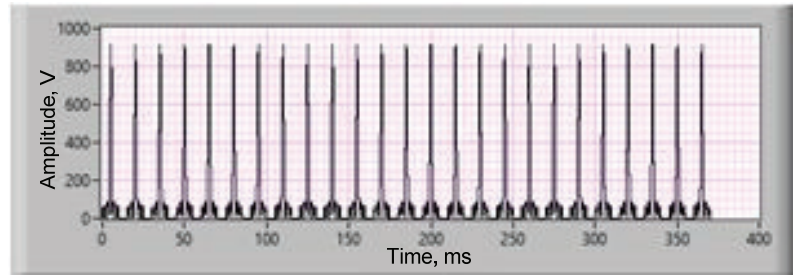

Fig. 5. Timing diagram of the envelope of the burst. 
The spectrum of packets of pulses after matched filtering is presented in figure 6 . This spectrum represents the spectrum of the linear frequency modulated signal.

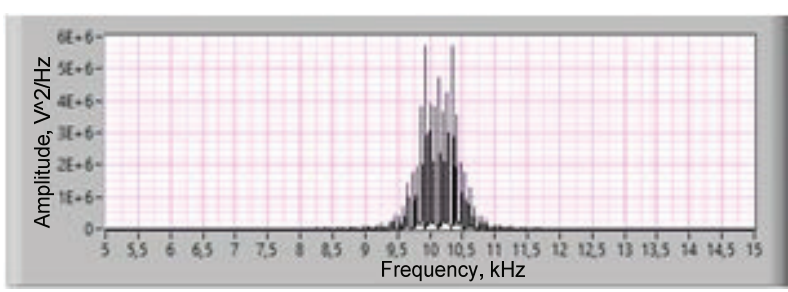

Fig. 6. The spectrum of the envelope of the burst.

The timing diagram accept packets of pulses is shown in figure 7.

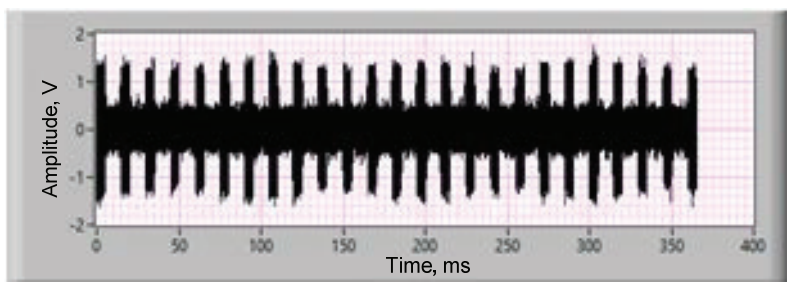

Fig. 7. Timing diagram of the receiving packet of pulses

In figure 7 one can notice the parasitic amplitude modulation caused by the rotating parts of the object. Figure 8 shows the spectrum of the received pulse packet.

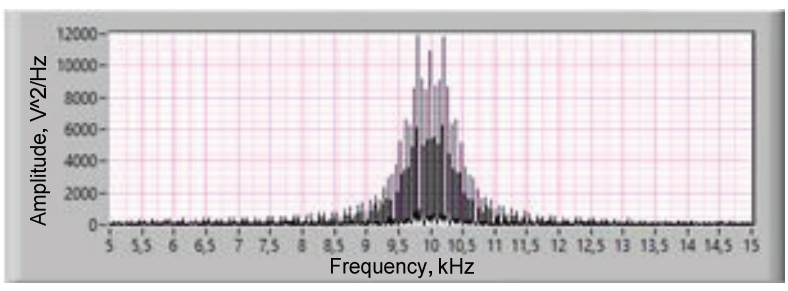

Fig. 8. The spectrum of the received burst of pulses

Further to the accepted signal is applied the procedure of matched filtering figure 9 and highlight the envelope of figure 10.

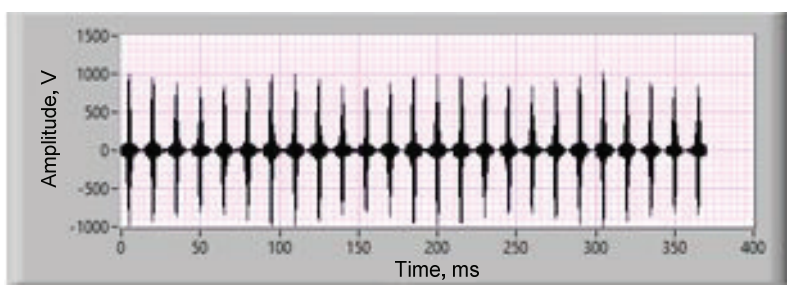

Fig. 9. Timing diagram of the received burst after consistent filtering.

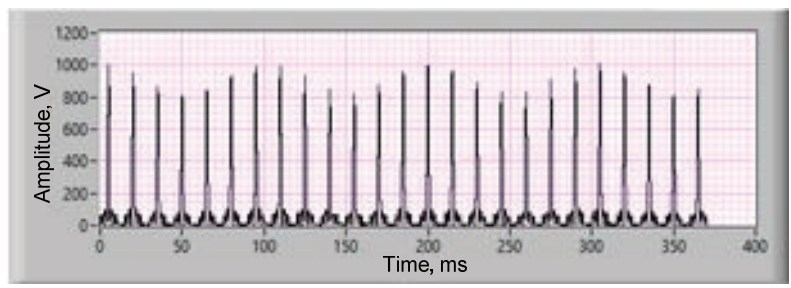

Fig. 10. Timing diagram of the envelope of the burst.

Then use the smoothing and reconstruction of a received signal. Due to the smoothing in the spectrum of the received signal remains one spectral component corresponding to the response signal from the aerodynamic object. The recovered spectrum of the signal shown in figure 11 .

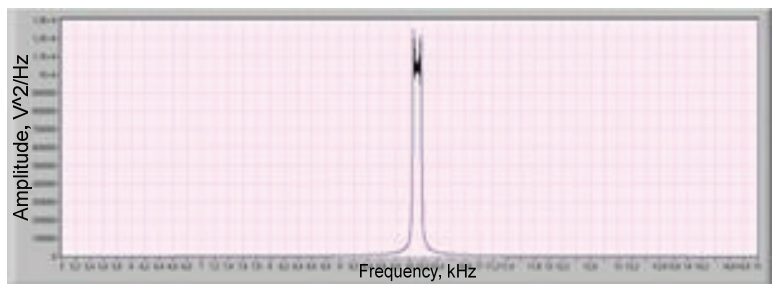

Fig. 11. Recovered signal spectrum.

When comparing the spectrum of the recovered signal (figure 11) and the spectrum of the received signal (figure 8 ), the reconstructed signal is smoothed by a parasitic modulation, which is a consequence of the presence of the turboprop component.

The study in the received signal contains only the useful component of the radio signal from aerodynamic facility, which is a body component. As a result of applying the proposed techniques were able to eliminate the noise components of the signal and interfering components, caused by the presence of turbo-propeller effect of the rotating parts of the aerodynamic object.

On the basis of the obtained results shows the effectiveness of smoothing algorithm on the proposed turboprop component models of the radiated radar signal.

\section{References}

1. E.O. Evdokimova, V.P. Fedosov, In Proc. International Scientific Conference "Emission and scattering of EMV - IREMV-2-13". - (2013)

2. E.O. Evdokimova, Izvestiya SFU. Technical science, no. $5,(2013)$

3. A.V. Kucheryavenko, In Proc. VII International Scientific and Practical Conference, no.2 (2015)

4. A.V. Kucheryavenko, V.P. Fedosov, In Proc. XII AllRussian Scientific Conference of Students, PostGraduates and Young Scientists "Information Technologies, Systems Analysis and Management (ITSAiU-2014)" 192 p. (2014) 